\title{
Research on the Connectivity of Ad-Hoc Networks Based on three- dimensional Node Distribution Network Model
}

\author{
Bi Xiaoming*
}

Wenzhou Vocational College of Science and Technology, Wenzhou, 325006, China

\begin{abstract}
In order to solve the problems in complicated connectivity analysis and low accuracy of the three-dimensional Ad-Hoc network, a new model, namely three-dimensional node distribution network model, is established for describing the structure of Ad-Hoc network and communication between nodes in three-dimensional. The new model realistic simulates the state and state changes of Ad-Hoc network. Based on the analysis by the proposed model, the relative motion of any node not only reduces the requirement of the communication range of any note, but also has the ability to enhance the connectivity of the network. Moreover, in contrast to the non-pause network, the connectivity of the pause network is in line with the network with uniform distribution.
\end{abstract}

Keywords: Ad-Hoc network, connectivity analysis, three-dimensional node distribution mode, network model, network connectivity, pause network, non-pause network.

\section{INTRODUCTION}

In the static organized sensor network, the node of the network was static stable arranged namely the location and the connection relationship of every node were stable. Hence, in the case of no-node failure, the connection of the network would not change a lot. However, in the mobile Ad-hoc network, owing to the relative movement of every node, the topology structure of the network would change significantly. Comparing with static wireless self-organized sensor network, the connection characteristic between the relative movement of node and network were depended on the following aspects:

(1) The dynamic change of the link. When mobile node turned into the weak connectivity of the network area, this node would be established for the isolated network node from connecting link, reduced the burden of key nodes of the business, hence, not only can improve the connectivity of network and the network capacity will also be able to balance the network energy consumption. On another hand, due to the movement of nodes, the node could be made beyond the scope of other nodes communication and become inaccessible nodes or isolated nodes. Finally, in the process of data forwarding, nodes moved easily disrupted communications link.

(2) Increasing of the network's consumption. Wireless self-organized network operation and maintenance of the need to constantly to obtain the state of the network, especially in the routing information of the network (e.g. in the case of high-speed mobile nodes and network topology changes), the effective time of the routing information is particularly short, in order to maintain network run efficiently, the network needed to constantly change the detection node link and update the current routing information.

(3) Influence of the locally connected network performance. In the different mobile model, the movement of the network node differenced obviously, its impact on network connectivity was also different.

Based on the above analysis, the 3D mobile selforganized network connectivity analysis of the complexity of existing in current studies will be real 3D simplified as twodimensional network and even the phenomenon of one dimensional network, with ignoring the necessary space information it would lead to the reliability of analysis results which was decreased obviously. For this defect, this paper utilized simulation method, under the constant in the threedimensional space, from two aspects of synthesis movement model and the trajectory model to discuss mobile selforganizing network connectivity model.

\section{3D MOBILE WIRELESS SELF-ORGANIZED NETWORK MODEL}

Bounded Connected Link Model. In the mobile selforganized network, when assumed the period is $t$, the distance from network node $i$ to $j$ is $r_{i j}^{t}$, according to the bounded connected theory, the probability of two node in period $t$ is:

$$
p\left(r_{i j}^{t}\right)=1\left[r_{i j}^{t}<\tau\right] h_{\tau}(r), \quad r \geq 0
$$

In the equation (1), the communication scope $\tau$ is depended on the transmitting power $P_{t}$, and $h_{\tau}(r)$ is the func- 
tion of the influence of communication environment and node movement to the wireless link, which could be wrote as:

$$
h_{\tau}(r)= \begin{cases}1 & r=0 \\ \in(0,1) & 0<r<\tau \\ 0 & \tau \leq r \leq 1\end{cases}
$$

3D Wireless Node Moving Model: In the research of mobile wireless self-organized network, the characteristics of moving node could be presented by mobile model. According to the modeling methods, the model could be divided as moving trajectory model and synthetic model. Among them, the moving trajectory model is based on mobile node trajectory tracking measurement; get the trajectory file, in the process of network analysis and simulation envoys point according to the moving trajectory. Is to point to in the process of simulation and synthesis of mobile model parameters according to the location of the nodes in the past time, according to a certain real-time calculate and determine the location of nodes of the current moment. The synthetic mobile model should be close to true as far as possible to the movement of mobile nodes, the accuracy of the approximation of the has important influence on the results of network analysis.

At present, there are dozens of mobile model of node, the more [1-3], which were widely applied in the study of selforganization network: Bettstetter [3] from mobile space dimension of the model, the degree of detail level, application field and random node of the aspects of mobile model are classified. In the existing mobile node model, a peacekeeping dominated the mobile model of two-dimensional, threedimensional scene node under the boom is only just emerging mobile model. Tai Suk [4] and others according to indoor environment, this paper proposes a three-dimensional high-rise building indoor mobile model, simulate the movement of the high-rise building my wife. Broyles [5] and others for high dynamic network, this paper puts forward a three-dimensional Gauss-Markov model (GM) mobile, Rohrer [6] and so on. On this basis, the 3-D Smooth Gauss-semiMarkov mobility model (3-D SGM) was put forward by Zheng Bo [7], the link dynamics of the wireless ad hoc networks was analyzed, the link breakage probability and the expected link life time between two nodes was solved, and then, for the three-dimensional constant velocity model [8], the link lifetime expectation, link formation rate and link breaking rate were calculated.

In the 3D random node moving model, the network node in a $3 \mathrm{D}$ space is harmonic separated and each node obeys the following rule from $W_{i}$ to $W_{i+1}$ :

(1) Node $j$ in the current position $W_{i}$ for a period of time $t_{p i}, t_{p i}$ obey uniform distribution in $\left[t_{\min }, t_{\max }\right]$.

(2) Node $j$ from the 3D area A randomly selected from A road as the target position $W_{i+1}$, the choice of $W_{i+1}$ has nothing to do with the nodes of the current and historical position;
(3) Node $j$ obeyed the uniform distribution to select velocity $v_{i} \in\left[v_{\min }, v_{\max }\right]$ to the next node $W_{i+1}$.

(4) Node $j$ would repeated the above route when arrived at node $W_{i+1}$.

Using synthetic mobile model in network simulation analysis to bring great convenience, at the same time there are also affect the characteristics of network simulation results: (1) The boundary effect: in a moving boundary node model, the node movement occurs only in a limited range, when the mobile node to the border of the region, its moving direction are influenced by the boundary, no longer subject to uniform distribution, the existence of this phenomenon will lead to inaccurate network analysis and simulation. (2) The average velocity attenuation in RWP mobile model, the average speed of all nodes in the network gradually increased with the increase of the simulation time is reduced, namely all nodes in the network will be stationary, to degenerate into a static network.

Aiming at these problems, on the basis of bounded connected link model, this paper established the following 3D mobile wireless self-organized network model:

(1) The spatial distribution of network nodes: K node independent random distribution in the three-dimensional space range $\mathrm{A}$;

(2) Bounded connecting link between the network nodes: assume that the network nodes with the same radius of communication within $\tau$ and the Omni-directional antenna, establish direct wireless link between any two nodes of probability to meet $p\left(r_{i j}\right)=1\left[r_{i j}<\tau\right] h_{\tau}(r)$, where $r_{i j}$ for the node of the Euclidean distance between nodes i and $\mathrm{j}, h_{\tau}(r)$

is the influence of environment on the node communication function, and only by the two-way communication link between nodes;

(3) Node mobility: (i) The three dimensional random waypoint model; Set node movement speed range and $\left[v_{\min }, v_{\max }\right]$ waypoint $\left[t_{\min }, t_{\max }\right]$ residence time range, working hours in the network, the node according to the three dimensional random road model mobile with movement. (ii) Track mobile model; Obtained by observing the node movement file, working hours on the internet and mobile node according to the trajectory data.

\section{3D MOBILE WIRELESS SELF-ORGANIZED NETWORK CONNECTIVITY SIMULATION ANAL- YSIS}

3D RWP Moving Network Connectivity: For the three dimensional random waypoint model of mobile network connectivity, analysis of different mobile parameters by using the method of simulation and the node communication range under the condition of network connectivity changes, related network connectivity evaluation parameters mainly include: 


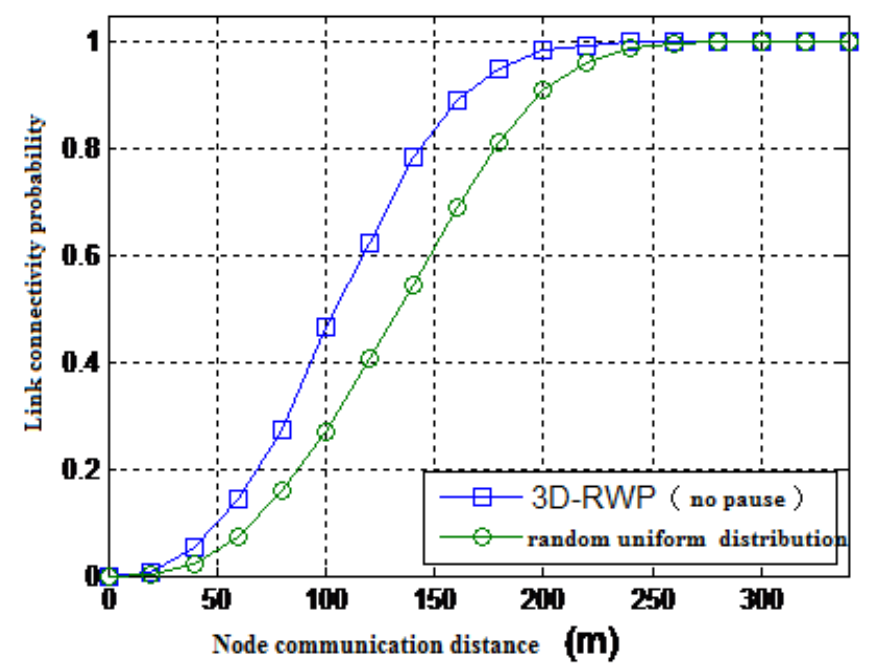

Fig. (1). RWP compared with uniform distribution network link connectivity probability model.

Link Connectivity Probability: Changing over time distance between two nodes of $r_{t}$, according to the bounded connected model, $t$ times the probability of two nodes to establish a communication link to $p\left(r_{t}\right)=1\left[r_{t}<\tau\right] h_{\tau}(r)$. Set times for $N$ mobile model simulation, simulation time is $T$, single simulation nodes connected in time for the $T_{i}$, is in a three-dimensional RWP mobile model, the average link connectivity probability for any two nodes:

$p_{\text {RWPlink }}=p\left(r_{t}\right) \approx \frac{1}{N} \sum_{i=1}^{N} \frac{T_{i}}{T}$

High Probability Connection: Set network node number is $K, t$ moment number of nodes in the network's largest connected component for $C_{t}$, is the biggest network $t$ time component ratio for:

$\lambda_{t}=\frac{C_{t}}{K}$

Largest connected component proportion said has the maximum number of nodes connected subnet accounted for the proportion of the whole network, it reflects the overall network connectivity. Called when $\lambda=1$ network to all connected; for large-scale networks, when $\lambda \geq 90 \%(90 \%$ is approximation), connected network could be called a high probability.

A number of simulation for $N$, single simulation time for $T$, node communication range for $\tau$, simulation of a single network of more than $90 \%$ nodes interconnected $(\lambda>90 \%)$ for $t_{i}(\tau)$, the probability of connected network with high probability is:

$p_{\text {con }}=p(\lambda>90 \%) \approx \frac{1}{N} \sum_{i=1}^{N} \frac{t_{i}(\tau)}{T}$
Critical Communication Range: When more than 90\% nodes in network interconnected probability of 0.9 , namely, when $p(\lambda>90 \%)=0.90$ node average communication range can be used as a range of network high probability interconnected critical communication:

$C T R_{h}=\tau[p(\lambda>90 \%)=0.9]$

Due to the three dimensional random waypoint mobile network node steady-state distribution is a kind of nonuniform distribution of stationary process and RWP model is a kind of time [9], the traditional method of probability and stochastic process analysis is very complex, even is not workable. In this article, through the simulation method to analyze the three-dimensional RWP model of mobile network connectivity, the simulation and experimental results are as follows.

\section{SIMULATION ANLAYSIS}

Three-dimensional simulation on RWP mobile network connectivity:

No pause the connectivity of the network. Without a pause RWP networks refer to those nodes in each waypoint pause time $t_{p i}$ is 0 , namely node in the process of moving not pause. Due to the uniform distribution network node requires movement over a period of time to reach steady state distribution of literature [1], this paragraph of time the experience value is given for more than $1000 \mathrm{~s}$, this paper set up the network simulation in the experiment before the $3600 \mathrm{~s}$ to ignore phase, namely only take $3600 \mathrm{~s}$ after starting the simulation data, the use of mobile model simulation tools for Bonnmotion [10].

In Fig. (1), the pause 3D RWP model of network link connected is adopted under the condition of uniform distribution probability and node link connectivity probability. 


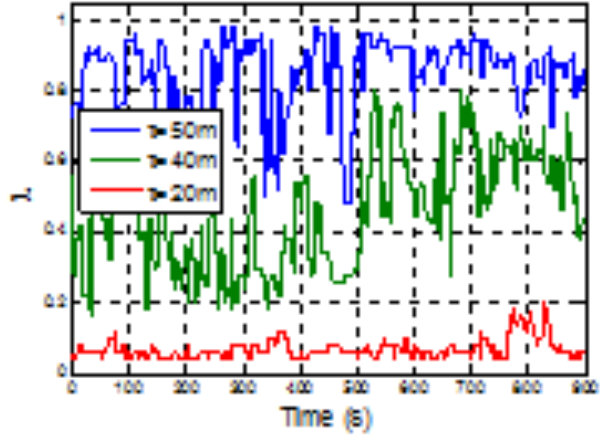

$\mathrm{K}=50$

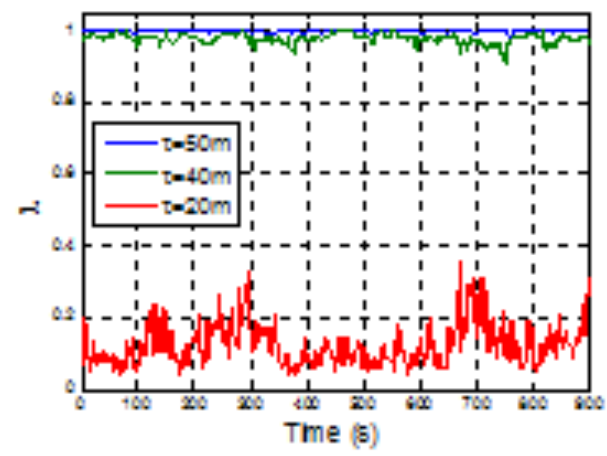

$\mathrm{K}=200$

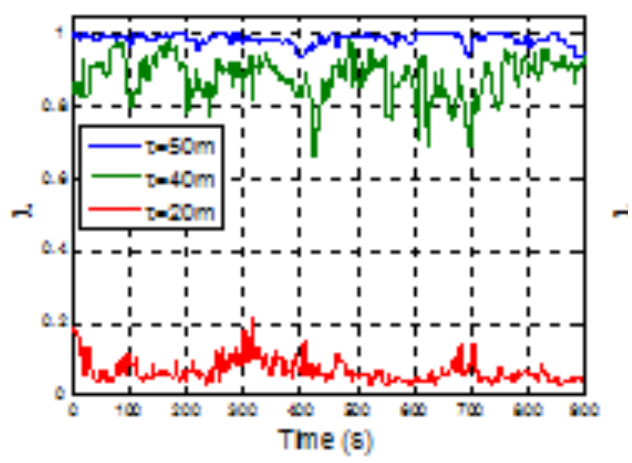

$\mathrm{K}=100$

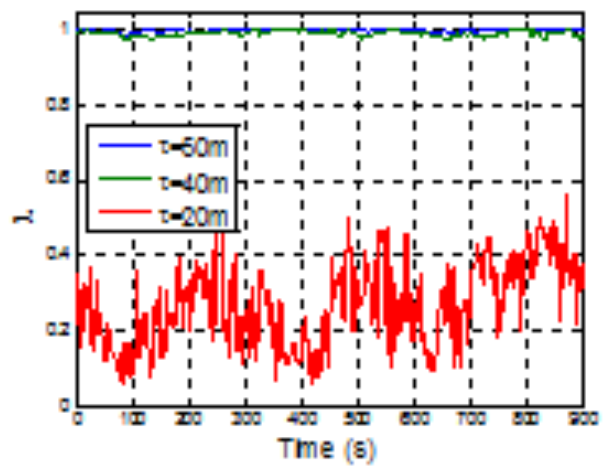

$\mathrm{K}=300$

Fig. (2). Mobile network connectivity dynamically.

The speed of the spread network $200 \times 200 \times 200\left(\mathrm{~m}^{3}\right)$ is $v$, the node speed $v$ obey uniform distribution $[0.5 \mathrm{~m} / \mathrm{s}, 1.5 \mathrm{~m} / \mathrm{s}]$, and $t_{p i}=0 \mathrm{~s}$ is the stay time. The intervals of sample experiment take $N=100, T=9000 s, t_{s}=1 s$, and the simulation results is the average of the $9 \times 10^{5}$ time network topology. Can be seen from the figure, compared with the uniform distribution of network nodes, under the condition of the same link connected probability, the $3 \mathrm{~d}$ - RWP required communication model of mobile node distance, the smaller the link connectivity is better. For example, when $p(r)=0.9, r_{R W P}=163 m, r_{\text {uniform }}=198 m$, it also reflects no pause 3D RWP steady non-uniform distribution of the model.

Shown in Fig. (2), the different network scale and the node communication scope of cases, the three dimensional RWP mobile network connected component proportion biggest change over time as a result, the network range for $200 \times 200 \times 200\left(\mathrm{~m}^{3}\right)$ scene. Experiment simulation time $T=9000 \mathrm{~s}$, sampling interval $t_{s}=1 \mathrm{~s}$, node number of 50 , 100,200 and 100 respectively. It can be seen that due to the mobile node, network connected component proportion biggest dynamic change over time; And, with the increase of network scale, the largest connected component proportion $\lambda$ gradually approaching to 1 , the asymptotic fully connected network.

As shown in Fig. (3), the different network scale and the node communication scope of cases, the connected network with high probability (more than $90 \%$ nodes) probability distribution. Experimental simulation times $N=10^{3}$, single simulation time for $T=900 \mathrm{~s}$, network range for $200 \times 200 \times 200\left(\mathrm{~m}^{3}\right), v$ node speed obey uniform distribution of $[0.5 \mathrm{~m} / \mathrm{s}, 1.5 \mathrm{~m} / \mathrm{s}]$, residence time $t_{p i}=0 \mathrm{~s}$. Experimental results show that the scope of node communication and network scale (node density) are the key factors influencing the network connecting high probability, when $p(\lambda>90 \%)=0.90$ node communication range can be used for critical communications network nodes, namely $C T R_{h}=\tau[p(\lambda>90 \%)=0.9]$, as you can see from the picture, under the condition of the simulation, the node number of 50 , 100 , and 200 3D RWP mobile network of critical communication range of $r_{c 1}=58 \mathrm{~m}, r_{c 2}=45 \mathrm{~m}$ and $r_{c 3}=36 \mathrm{~m}$ respectively. At the same time, also you can see that the network connectivity probability from sharp transition phenomenon between 0 and 1 , and the node number, the greater the sharp transition phenomenon is more obvious. 


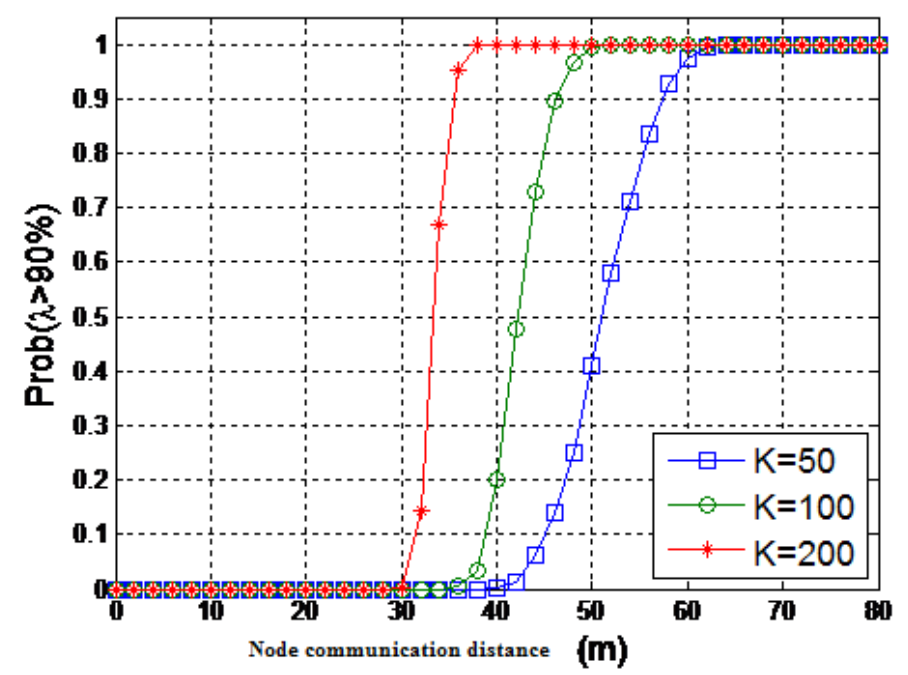

Fig. (3). Network connecting the probability distribution of the high probability.

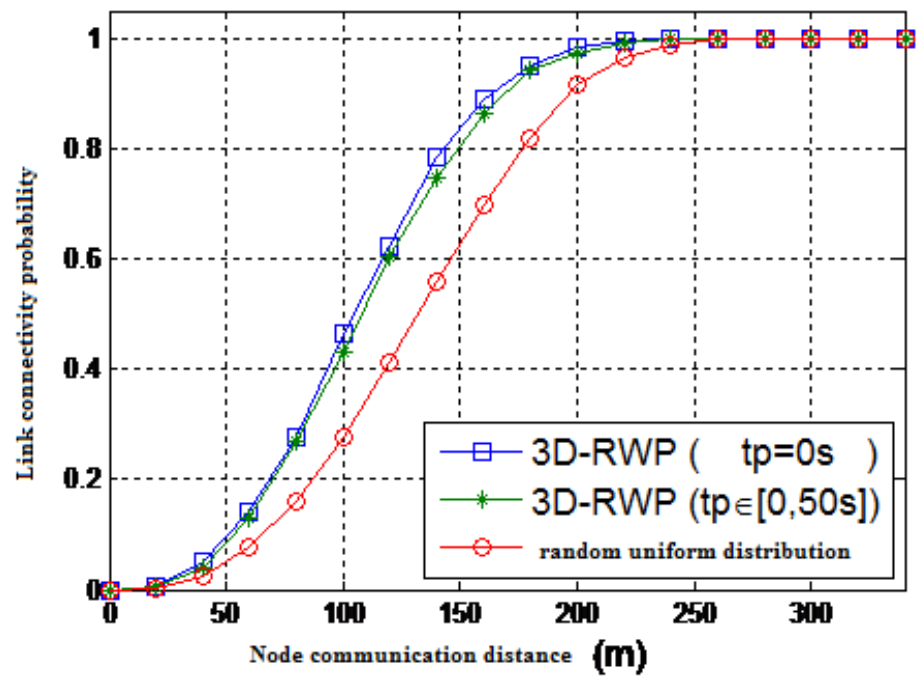

Fig. (4). Three-dimensional RWP network link connectivity probability.

Pause the connectivity of the network. Pause threedimensional RWP mobile model in network, the node in the retention period of way points distributed evenly in the $t_{p i}$ to $\left[t_{\min }, t_{\max }\right]$. As is shown in Fig. (4), Network range for $200 \times 200 \times 200\left(\mathrm{~m}^{3}\right)$, node speed obey uniform distribution $[0.5 \mathrm{~m} / \mathrm{s}, 1.5 \mathrm{~m} / \mathrm{s}]$, and $v$ time $t_{p i}$ obey uniform distribution within the $[0,50 s]$, number of $N=100, T=9000 \mathrm{~s}$ single simulation time, sampling interval $t_{s}=1 \mathrm{~s}$. Can be seen from the figure, compared with no pause network, have a pause network link connectivity probability is lower, namely as network mobility increases, the probability of nodes connected. At the same time, according to the analysis of the link connected probability, it can further the node degrees and the total probability of the network.
As shown in Fig. (5) under different network scale and the node communication range, pause connected network with high probability of a probability distribution. Network range for $200 \times 200 \times 200\left(\mathrm{~m}^{3}\right), v$ to $[0.5 \mathrm{~m} / \mathrm{s}, 1.5 \mathrm{~m} / \mathrm{s}]$ node speed of uniform distribution, the residence time $t_{p i}$ obey uniform distribution of different range simulation frequency $N=10^{3}, T=900 \mathrm{~s}$ single simulation time, network node number of $K_{1}=50, K_{2}=100$ and $K_{3}=200$, respectively. By the simulation results can be obtained, different scale network connected $\mathrm{k}$ high probability $(p(\lambda>90 \%)=0.9)$ is the critical range of communication. Critical communication range when $t_{p i} \in[0,50 \mathrm{~s}], \quad r_{c 1}=60 \mathrm{~m}, r_{c 2}=47 \mathrm{~m}$ and $r_{c 3}=37 \mathrm{~m}$, respectively; Critical communication range when 


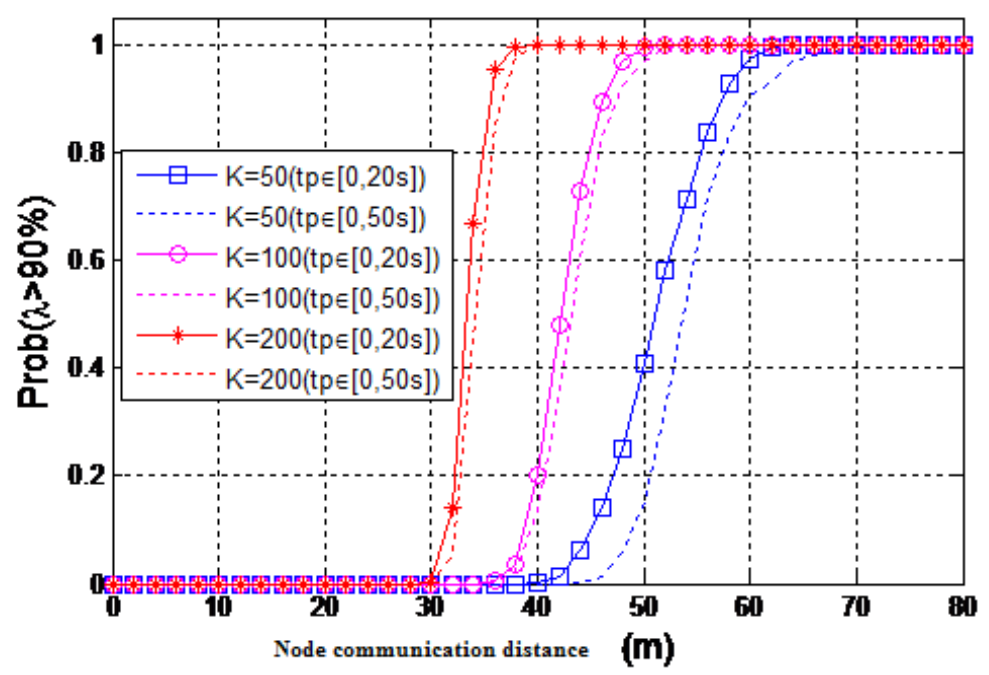

Fig. (5). Network connecting the probability distribution of the high probability.

$t_{p i} \in[0,20 s], \quad r_{c 1}^{\prime}=57 m, r_{c 2}^{\prime}=46 m$ and $r_{c 3}^{\prime}=36 m$ respectively. Hence, the stronger the network node mobility, the smaller network of critical communication ranges. Therefore, mobile network in terms of average node connectivity probability is greater than the static network, in short connection communication performance is better, but high-speed mobile, leading to the link between the nodes to maintain time is shorter, not suitable for long connection.

\section{CONCLUSION}

This paper proposes a new mobile organization can be described in three dimensional space network status and the change of state of network model. Based on the model of 3D mobile wireless self-organized network connectivity is analyzed. Analysis and experiment results show that the three dimensional random waypoint mobile self-organizing networks, exercise can reduce the requirement of network of node communication range, improve the ability of network connectivity; Pause the network and compared with seamless network, the network connectivity is closer to uniform distribution network.

\section{CONFLICT OF INTEREST}

The author confirms that this article content has no conflict of interest.

\section{ACKNOWLEDGEMENTS}

Declared none.

\section{REFERENCES}

[1] T. Camp, J. Boleng, and V. Davies, "A survey of mobility models for ad hoc network research," Wireless Communication Mobile Computing, vol. 2, no. 5, pp. 483-502, 2002.

[2] A. Jardosh, E.M.B. Royer, K. Alemroth, and S. Suri "Towards realistic mobility models for mobile ad hoc networks," In: Proceedings of the $9^{\text {th }}$ Annual International Conference on Mobile Computing and Networking, 2003, pp. 217-229.

[3] C. Bettstetter, "Mobility modeling in wireless networks: categorization, smooth movement, and border effects," Mobile Computing and Communications Review, vol. 5, no. 3, pp. 55-66, 2001.

[4] S. Tai, K. Jae, and K. Dan, "Mobility modeling and traffic analysis in three-dimensional high-rise building environments," Vehicular Technology, vol. 49, no. 5, pp. 1633-1640, 2000.

[5] D. Broyles, A. Jabbar, and J. Sterbens, "Design and analysis of a 3D gauss-markov mobility model for highly-dynamic airborne networks," In: Proceedings of the International Telemetering Conference, pp. 201-206.

[6] J. Rohrer, E. Cetinkaya, H. Narra, D. Broyles, K. Peters, and J.P.G. Sterbenz, "AeroRP performance in highly-dynamic airborne networks using 3D Gauss-Markov mobility model," In: Proceedings of the Militrary Communications, 2011, pp. 834-841.

[7] B. Zheng, H. Zhang, and G. Huang, "Link dynamics in MANET based on 3-D SGM mobility model," Journal of Jilin University: Engineering and Technology Ed, vol. 42, no. 5, pp. 1315-1320, 2012.

[8] B. Zheng, H. Zhang, and G. Huang, "Link dynamics in threedimensional mobile Ad hoc networks," Journal of Electronics and Information Technology, no. 11, pp. 2605-2609, 2011.

[9] R. La, and E. Seo, "Network connectivity with a family of group mobility models," IEEE Transactions on Mobile Computing, vol. 11, no. 3, pp. 504-517, 2012.

[10] N. Aschenbruck, R. Ernst, E. Gerhards-Padilla, and M. Schwamborn, "BonnMotion: a mobility scenario generation and analysis tool," In: Proceedings of the $3^{\text {rd }}$ International Conference on ICST, 2010, pp. 206-301.

(C) Bi Xiaoming; Licensee Bentham Open.

This is an open access article licensed under the terms of the Creative Commons Attribution Non-Commercial License (http://creativecommons.org/licenses/by-nc/3.0/) which permits unrestricted, non-commercial use, distribution and reproduction in any medium, provided the work is properly cited. 